\title{
Rare Case of Asymptomatic Cardiac Metastasis in a Patient of Laryngeal Squamous Cell Carcinoma
}

\section{Ayman Hassan Elshiekh $^{1 *} \mid$ Abdelrahman Elhadary ${ }^{2} \mid$ Waad Fuad Bin Afif ${ }^{3}$}

*Correspondence: Ayman Hassan Elshiekh

Address: ${ }^{1}$ Department of Cardiology, Ministry of the National Guard- Health Affairs, Jeddah, Saudi Arabia. King Abdullah International Medical Research Center, Jeddah, Saudi Arabia. King Saud Bin Abdulaziz University for Health Science, Jeddah, Saudi Arabia; ${ }^{2}$ Department of Cardiology, Ministry of the National Guard- Health Affairs, Jeddah, Saudi Arabia; ${ }^{3}$ Department of Medicine, Ministry of National Guard-Health Affairs, Jeddah, Saudi Arabia

e-mail $₫$ ay_elshiekh@yahoo.com

Received: 15 March 2021; Accepted: 19 March 2021

Copyright: (C) 2021 Elshiekh AH. This is an open-access article distributed under the terms of the Creative Commons Attribution License, which permits unrestricted use, distribution, and reproduction in any medium, provided that the original work is properly cited.

\section{ABSTRACT}

Background: Cardiac tumors are relatively uncommon and mostly secondary to primary tumors in the lung, esophagus, breast, lymphoma and melanoma. Most of these tumors are detected incidentally as they are usually clinically silent. We present a rare case of laryngeal cancer with asymptomatic myocardial metastases.

Case Report: A 71-year-old man known case of metastatic laryngeal squamous cell carcinoma on tracheostomy tube due to airway compromise and on chemotherapy, was admitted to the oncology department due to aspiration pneumonia. The patient underwent routine chest X ray and an echocardiogram with contrast that revealed a small mass attached to the apical part of left ventricle (LV) lateral wall with normal contractility of adjacent myocardium then Cardiac MRI was done and confirmed the presence of cardiac metastases. This is one of the few reported cases of laryngeal carcinoma with LV metastases.

Conclusion: Cardiac metastases should be considered in priority of differential diagnosis of any cardiac mass in a patient with underlying malignancy.

Keywords: Cardiac Metastases, Laryngeal Cancer, CMR

\section{Case Presentation}

Tumours metastatic to the heart (cardiac metastases) are among the least known and highly debated issues in oncology, and few systematic studies are devoted to this topic. A 71-year-old man was initially diagnosed with laryngeal squamous cell carcinoma and treated with chemotherapy. He was admitted in oncology department for progressive shortness of breath and productive cough where he was diagnosed as aspiration pneumonia associated with pleural effusion. He was treated with broad spectrum antibiotics and pleural drain. Initially his condition improved. Routine echocardiogram revealed normal biventricular dimension and systolic function with no significant valvular diseases, but 
an accidental small mass attached to anterolateral wall of left ventricle was noted and protruded into the left ventricular cavity with normal contractility of adjacent myocardial segments. This raised the possibility of cardiac metastases and less likelihood of thrombus. However, we confirmed the presence of the mass by a contrast echocardiogram study.

Cardiac magnetic resonance (CMR) study was done, and it confirmed the presence of a single mass attached to the anterolateral wall of the left ventricle typical for metastases (Fig. 1-3).

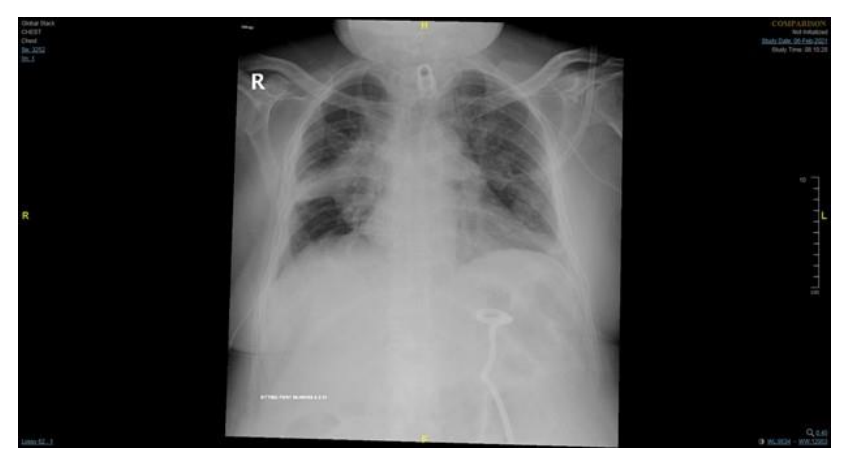

Figure 1: Chest $x$ ray of the patient

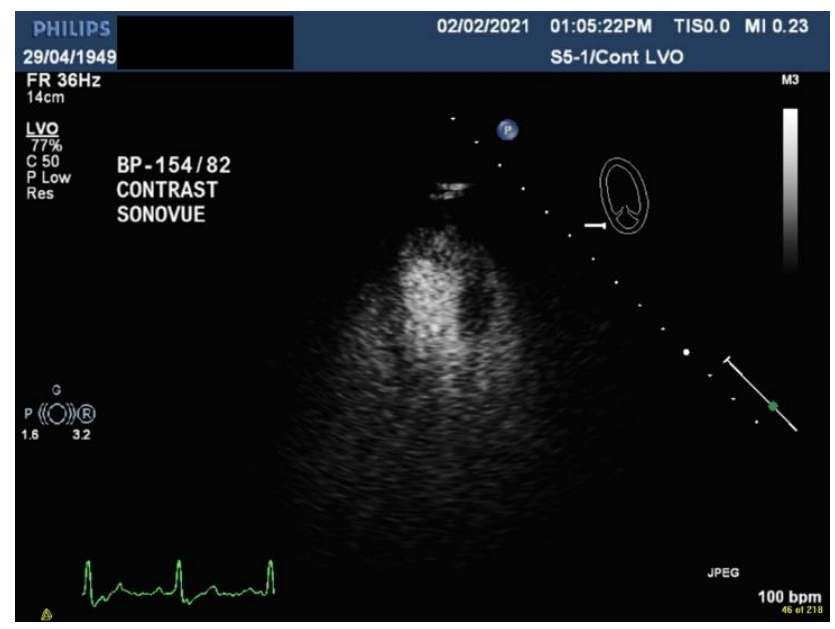

Figure 2: Cardiac echocardiography with contrast showing the mass

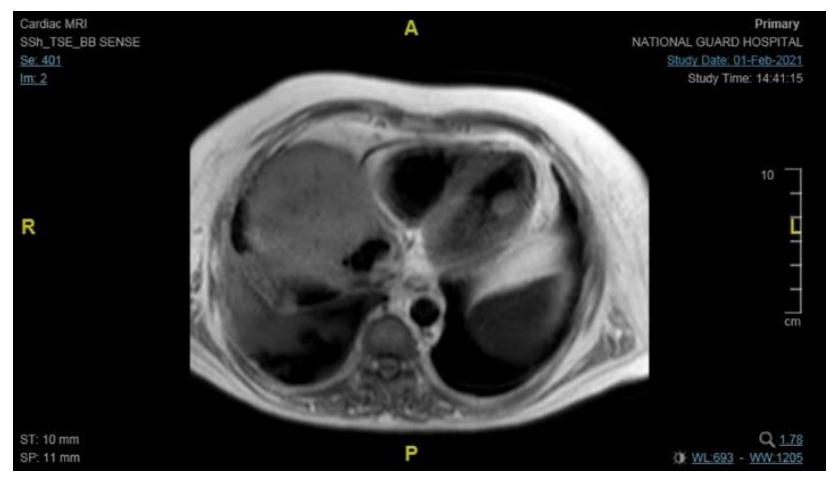

Figure 3: Cardiac MRI show the mass in the left ventricle same as in echo 


\section{Discussion}

According to the literatures, Metastatic cardiac tumors (MCTs) involving the myocardium and pericardium are rare (Bruce, 2011), with reported incidence of 1.5-20\% of autopsies of cancer patients. Although cardiac metastases (CM) are much more common than primary cardiac tumors, the diagnosis of CM antemortem is seldom made, because more than $90 \%$ are clinically silent. About $75 \%$ of primary cardiac tumors are benign and 25\% are malignant (4\% primary malignancy and $96 \% \mathrm{CM}$ ) (Hoffmeier et al., 2014). Though these metastases may originate from any malignant tumor, they are most commonly caused by melanoma, lymphoma, leukemia and carcinoma of the lung, breast and esophagus (Burazor et al., 2018). There have been only few reported cases of laryngeal cell carcinoma with cardiac metastases in living patients. The interesting in our patient also that he presented with myocardial metastases in the left ventricle which is considered less common than other cardiac sites for metastases like pericardium.

The occurrence of cardiac metastasis is as the following in order of frequency, about two thirds of all cardiac metastases involve the pericardium (69.4\%), one third the epicardium (34.2\%) or the myocardium (31.8\%) and only 5\% the endocardium (Bussani et al., 2007).

Metastatic tumours can spread to the heart through multiple routes (Burazor et al., 2018):

1. Hematogenous: seen in melanoma, lymphoma and sarcoma and associated with myocardial and endocardial metastases.

2. Lymphatic: that characteristic for lung carcinoma and results in pericardial and epicardial involvement.

3. Transvenous: typical for renal cell carcinoma, hepatocellular carcinoma, leiomyoma of the uterus and pheochromocytoma that extends through inferior vena cava into the right atrium.

4. Direct: encountered in locally aggressive tumors such as mediastinal and pleural tumors as well as carcinomas of the breast.

Clinical presentations of cardiac metastases are extremely variable. Although a cardiac metastasis may be the first or even the only manifestation of an undiagnosed malignant neoplasm, they often go unrecognized in vivo and are diagnosed only after death (Tandon et al., 2019). In general, although a focal lesion secondary to the myocardium may result in unclear symptoms that may go undetected, tumors spreading more extensively to the pericardium or to other cardiac sites may produce dramatic clinical patterns, causing medical emergencies (Kim et al., 2019). The most typical scenario would be a patient with lung or breast cancer presenting with increasing shortness of breath, hypotension and 
tachycardia and clinical findings suggestive of tamponade. Neoplastic pericardial effusions are indeed among the most feared complications. Although they may be mild and result in no symptoms, they are commonly symptomatic and often the ultimate cause of death. Due to the wide range of clinical presentations in patients with cardiac metastases, detection is often incidental (Bussani et al., 2007). Echocardiography is an ideal initial imaging modality since it is simple, non-invasive, widely available, and low cost with accuracy has been reported to be as high as $80 \%$. It delineates the morphologic appearance, location and motion of tumors as well as determining the hemodynamic consequences of the tumor, if any. CT and MRI are complimentary techniques that provide additional diagnostic information and are useful for staging and treatment planning, particularly when surgical resection is being considered. CT has better spatial resolution than MRI and also provides information about vascularity and calcification. MRI also provides information about vascularity, has better overall tissue characterization, and does not have the attendant risk of radiation. There is no standard therapeutic option for patients with cardiac metastases and because of the multi-modalities of treatment, a multidisciplinary team of cardiologist, oncologist and cardiothoracic surgeon, working with the patient, should contribute to the therapeutic decision-making process.

\section{Summary}

Metastatic cardiac tumours are rare with about 0.5-2 \% cardiac involvement. Most of these metastases came from lung, breast, melanoma, lymphoma and leukemia. These metastases rarely diagnosis antemortem. Our case is a very rare laryngeal cell carcinoma which sent metastasis to the heart and accidently discovered by echo. No specific therapies recommended to such a kind of metastasis to the heart.

\section{Reference}

Bruce CJ. Cardiac tumours: diagnosis and management. Heart 2011; 97: 151-160.

Burazor I, Aviel-Ronen S, Imazio M, Goitein O, Perelman M, Shelestovich N, Radovanovic N, Kanjuh V, Barshack I, Adler Y. Metastatic cardiac tumors: from clinical presentation through diagnosis to treatment. BMC Cancer 2018; 18: 1-9.

Bussani R, De-Giorgio F, Abbate A, Silvestri F. Cardiac metastases. Journal of Clinical Pathology 2007; 60: 27-34.

Hoffmeier A, Sindermann JR, Scheld HH, Martens S. Cardiac tumors--diagnosis and surgical treatment. Dtsch Arztebl Int 2014; 111: 205-211.

Kim JK, Sindhu K, Bakst RL. "Cardiac Metastasis in a Patient with Head and Neck Cancer: A Case Report and Review of the Literature". Case Reports in Otolaryngology 2019; 2019: 8.

Tandon V, Kethireddy N, Balakumaran K, Kim AS. Metastatic squamous cell carcinoma to the heart: an unusual cause of ST elevation-a case report. European Heart Journal-Case Reports 2019; 3: ytz029. 